\title{
Where Memory Meets Attention: Neural Substrates of Negative Priming
}

\author{
Tobias Egner and Joy Hirsch
}

\begin{abstract}
The negative priming (NP) effect refers to the observed increase in identification time for a current target stimulus or stimulus feature (the "probe") that has been employed as a distractor stimulus or stimulus feature on the previous trial (the "prime"), representing strong evidence that ignored information is actively processed to a high level by selective attention systems. However, theoretical accounts of NP differ in whether they attribute the effect to processes of selective inhibition or episodic memory retrieval. Here we derived neurophysiological predictions from the rival "selective inhibition" and "episodic retrieval" models of NP, and employed event-related fMRI in a color-naming Stroop task to assess neural responses to probe
\end{abstract}

\section{INTRODUCTION}

Selective attention refers to the ability to preferentially allocate perceptual or higher-order resources to goalrelevant attributes of our internal or external environment, resulting in enhanced processing of the selected, relative to unselected, attributes. Traditionally, selective attention has been described as an active process directly operating on the to-be-attended information, with unattended information being processed either only very rudimentarily (e.g., Treisman, 1969; Broadbent, 1958), or processed fully at the perceptual level, but not impinging upon response processes (e.g., Norman, 1968; Deutsch \& Deutsch, 1963). Over the course of the last 30 years, however, a large body of work has documented that unattended information may in fact be actively processed to a high level, such that information that is currently ignored can have substantial effects on the processing of subsequent stimuli. For instance, Neill (1977) demonstrated that the naming of the color hue of a color-word stimulus on a Stroop task (MacLeod, 1991; Stroop, 1935) is slowed if the current color had been employed as the distractor (i.e., the word stimulus) on the previous trial, in comparison to trials where current target and previous distractor stimuli are unrelated (see also Dalrymple-Alford \& Budayr, 1966). This

Columbia University, New York trials that were subject to either no priming or negative priming. Compared to no-priming probe trials, NP resulted in increased activation of the right dorsolateral prefrontal cortex, in a region which has been closely linked with episodic memory retrieval functions. NP was also accompanied by activation of the right thalamus, particularly the mediodorsal nucleus, which has been implicated in the pathophysiology of schizophrenia, a condition associated with diminished NP effects. Our results support the proposal that ignored stimulus information is fully encoded in memory, and that episodic retrieval, not selective inhibition, of such information affects selective attention performance on subsequent trials.

effect, where target identification is slowed when a distractor stimulus or stimulus feature on the preceding trial (the "prime") turns into the target stimulus or stimulus feature on the current trial (the "probe") has been termed "negative priming" (NP), and constitutes compelling evidence for active processing of distractors by selective attention mechanisms to an extent that can negatively affect performance (Tipper, 1985; Tipper \& Cranston, 1985; Neill, 1977, 1979). To elucidate the psychological determinants and neural mechanisms of $\mathrm{NP}$ is of crucial importance for our understanding of selective attention processes, both in healthy as well as in disordered cognition, as NP has proved an important paradigm in schizophrenia research, with schizophrenic patients displaying diminished NP compared to matched controls (e.g., MacQueen, Galway, Goldberg, \& Tipper, 2003; Laplante, Everett, \& Thomas, 1992; Beech, Powell, McWiliam, \& Claridge, 1989).

NP effects have been documented in a large variety of tasks, including target naming, target matching, target localization, and lexical decision paradigms (for reviews, see Fox, 1995; May, Kane, \& Hasher, 1995). The effect appears to operate at the level of semantic stimulus representation (Yee, 1991; Tipper \& Driver, 1988; Allport, Tipper, \& Chmiel, 1985; Tipper, 1985), as it is neither dependent on priming of a specific type of response (Neill, Lissner, \& Beck, 1990; Tipper, MacQueen, \& Brehaut, 1988), nor on particular physical stimulus features (Driver \& Baylis, 1993; Tipper \& 
Driver, 1988; Neill, 1977). Although a substantial psychological research literature on NP has accumulated, a detailed review of which lies beyond the scope of this article (for reviews, see Fox, 1995; May et al., 1995), the nature of the underlying mechanisms of NP remains contested (Tipper, 2001; Neill, 1997). Although early interpretations of NP were unanimous in assuming that it represented a consequence of active inhibitory processes operating on the distractor stimulus or stimulus features (Neill \& Westberry, 1987; Tipper, 1985; Tipper \& Cranston, 1985; Neill, 1977, 1979), rival theoretical accounts have since been put forward, resulting in at least two major models of NP, the "selective inhibition" account (Houghton \& Tipper, 1994) and the "episodic retrieval" model (Neill \& Valdes, 1992; Neill, Valdes, Terry, \& Gorfein, 1992). These models are briefly presented in turn, and neurophysiological predictions are derived from each of them for the purpose of the current experiment.

\section{Selective Inhibition}

The selective inhibition account of NP holds that mental representations of both attended (target) and ignored (distractor) stimuli or stimulus features are initially activated, but that shortly after attentional selection, representations of the ignored information (or their links to response mechanisms) are actively inhibited (Houghton \& Tipper, 1996; Houghton \& Tipper, 1994; Tipper \& Cranston, 1985). Therefore, if the probe trial requires responding to information that has been ignored on the prime trial, the inhibition associated with that previously ignored information has to be overcome, resulting in slowing of responses. For example, in a color-naming Stroop task, if the prime trial stimulus was the word RED in blue hue, semantic representations of RED would subsequently be inhibited, and if the probe trial consisted of the word GREEN in red hue, the correct response ("red") would still be subject to inhibition. Given that the colornaming process in the probe trial is thus disadvantaged in its competition with the prepotent word-naming process (see Cohen, Dunbar, \& McClelland, 1990), it would be expected that the level of response conflict arising between these processing streams should be enhanced, relative to incongruent trials that are not subject to NP. This assumption of increased response conflict between color- and word-naming processing on negatively primed probe trials leads to the neurophysiological prediction that these trials should be characterized by increased activation of the medial prefrontal cortex (MPFC), particularly the anterior cingulate cortex (ACC), relative to nonprimed probes, as ACC activity has been shown to reliably covary with levels of response conflict in Stroop-like paradigms (Kerns et al., 2004; Ullsperger \& von Cramon, 2001; Carter et al., 2000; Casey et al., 2000; Botvinick, Nystrom,
Fissell, Carter, \& Cohen, 1999; for a recent review, see Botvinick, Cohen, \& Carter, 2004).

\section{Episodic Retrieval}

An alternative account for NP has been formulated in terms of episodic memory retrieval effects (Neill \& Valdes, 1992; Neill, Valdes, et al., 1992), on the basis of Logan's Instance Theory of Automatization (Logan, 1988). Logan's model holds that task learning reflects a transition from the reliance on slow rule-based algorithmic processing to performance mediated by the reliance on episodic memory retrieval of previous instances involving a given stimulus (and associated representations and responses). This episodic retrieval supplies a potential shortcut to past solutions and can lead to automatization of performance (i.e., faster and less variable responses) (Logan, 1988). In the case of NP, however, the retrieval process proves to be detrimental, as the most recent (and salient) episode involving associates of the target feature on the probe trial (e.g., GREEN in red hue) is the prime stimulus where the current target feature served as the distractor (e.g., RED in blue hue) and was associated with a nonresponse. This retrieved episode would contain "nonresponse" information and be of no use as a shortcut to the correct response on the current trial, leading to additional retrieval operations in search for an episode more closely corresponding to the current stimulus. It is this need for retrieving additional information from episodic memory, which may even result in falling back on slow algorithmic processing, that is held to produce the slowed response times in NP (Neill, 1997).

Similar to the selective inhibition model, according to the episodic retrieval account, negatively primed probe trials could be (but do not have to be) associated with increased response conflict compared to nonprimed trials, as the "nonresponse" stimulus information may interfere with the correct response to the current trial, leading to a prediction of MPFC/ACC activation. In contrast to the selective inhibition model, however, the episodic retrieval account specifically predicts an increased involvement of memory retrieval processes on such trials and, by inference, increased activation in brain regions supporting retrieval processes. Human neuroimaging studies of episodic memory retrieval processes have implicated right prefrontal cortical regions in particular (for reviews, see Fletcher, Frith, \& Rugg, 1997; Buckner, Raichle, Miezin, \& Petersen, 1996). Within the right prefrontal cortex, it has further been suggested that the dorsolateral prefrontal cortex (DLPFC) supports the monitoring and evaluation of information retrieved from episodic memory (Rugg, Henson, \& Robb, 2003; Henson, Rugg, Shallice, \& Dolan, 2000; Henson, Shallice, \& Dolan, 1999; Fletcher, Shallice, \& Dolan, 1998; Shallice et al., 1994), whereas more posterior ventrolateral prefrontal cortex (VLPFC) supports the specification of 
retrieval cues (Rugg et al., 2003; Henson, Rugg, et al., 2000; Henson, Shallice, et al., 1999).

Given that behavioral experiments have arguably not produced unambiguous support for either of these NP models (Fox, 1995; May et al., 1995), and that these models actually make quite diverse predictions regarding the brain processes that should underpin the NP phenomenon, it is surprising that there has been a scarcity of studies investigating potential neural correlates of this effect. The only previous fMRI study of NP known to the authors contrasted blocks of NEG-P trials with blocks of incongruent Stroop trials (Steel et al., 2001), and reported a number of rather small (3-9 voxels) and diffuse foci of increased activation, including medial and bilateral frontal and parietal, as well as left temporal regions. Considering the nature of this study's design, with no behavioral data recorded and no possibility of excluding error trials, as well as a low number of subjects $(n=7)$, no firm conclusions can be drawn from these imaging data. A recent electroencephalographic (EEG) investigation (Mayr, Niedeggen, Buchner, \& Pietrowsky, 2003) has found positive-going event-related potentials (ERPs) in parietal scalp regions from around 300-600 msec poststimulus to distinguish between negatively primed and nonprimed auditory probe stimuli. The authors suggested that the topography, latency, and polarity of this ERP bears resemblance to the "old/new effect" thought to be related to episodic memory retrieval (Rugg \& Nagy, 1989), and that by inference their data could be interpreted as supporting the episodic retrieval model of NP. The goal of the current study was to further our understanding of the mechanisms underlying NP by testing neurophysiological hypotheses derived from NP models by means of event-related fMRI measures of neural activity in response to probe stimuli that are subject to either no priming or NP, during a color-naming Stroop task (see Figure 1 and Methods).

Unfortunately, in the timing of Stroop stimulus events, there exists an inherent tradeoff between optimizing the trial sequence for fMRI detection of taskrelated brain activation and attempting to maximize behavioral NP effects. NP exhibits an exponential decay function when the response-to-stimulus interval (RSI; the interval between response to the prime stimulus and the appearance of the probe stimulus) is varied unpredictably, with the effect being most pronounced at an RSI of 50-1000 msec, but potentially undetectable at RSIs of 2000 msec and above (Neill \& Valdes, 1992; Neill \& Westberry, 1987). It should be noted that the assumption of an exponential decay function does of course imply that NP, in principle, remains present at longer intervals even in the case of random RSIs, but that it may not be detectable statistically. Optimizing the detection of BOLD responses in a fast event-related fMRI design, on the other hand, requires random jittering of interstimulus intervals (ISIs) (Dale, 1999; Burrock, Buckner, Woldorff, Rosen, \& Dale, 1998) and an average ISI of at

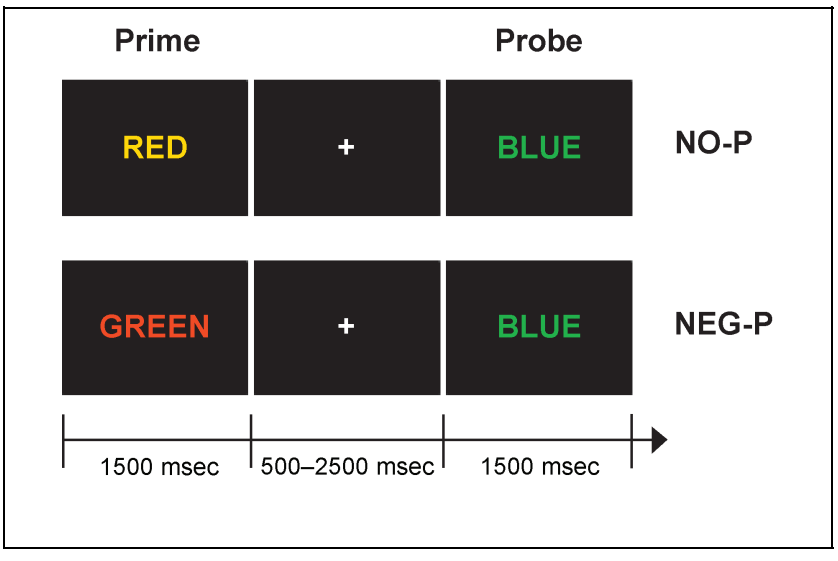

Figure 1. Example trials and timing parameters of the priming Stroop task, representing no priming (NO-P, top) and negative priming (NEG-P, bottom) trials. In NO-P trials, neither the target dimension (yellow ink color) nor the distractor dimension (the word meaning RED) are related between the prime and the probe stimulus. In NEG-P trials, the distractor dimension from the prime stimulus (the word meaning GREEN) becomes the target dimension of the probe stimulus (green ink color).

least 2000 to $4000 \mathrm{msec}$ (Wager \& Nichols, 2003), due to nonlinear interactions in BOLD responses between stimuli presented at faster rates (Vazquez \& Noll, 1998; Binder et al., 1994). It follows that stimuli presented at a rate likely to induce maximal behavioral NP would not easily be amenable to event-related fMRI analyses. As the primary purpose of this study was the identification of neural substrates of NP, our trial sequence was designed with the intention to optimize BOLD response detection, rather than maximizing behavioral NP effects. The use of a jittered ISI, however, enabled us to test a specific prediction of the episodic retrieval model of NP. According to this model, the likelihood of a previous stimulus instance to be retrieved on a given trial depends on the temporal discriminability (Baddeley, 1976) of that stimulus. Prime stimuli are maximally discriminable if they have a long preprime ISI and a short prime-to-probe interval, and least discriminable with a short preprime ISI and a long prime-to-probe interval (Neill \& Valdes, 1992; Neill, Valdes, et al., 1992). Therefore, NP probe trial reaction time (RT) should be longer on trials with a short prime-to-probe interval relative to the preprime ISI than on trials with a long prime-to-probe interval relative to the preprime ISI, and no such differences should be evident for no-priming trials. The inhibition model, on the other hand, would not predict NP to be affected by the interval between the probe stimulus of the previous trial and the prime stimulus of the current trial.

\section{RESULTS}

\section{Behavioral Data}

The Stroop task produced a significant interference effect on RT (interference $=48 \mathrm{msec}$ ), as responses 
Table 1. Mean Reaction Times (RT) and Percentage of Accurate Responses (\% Accuracy) with Respective Standard Deviations $(S D)$ for Probe Trials

\begin{tabular}{lcccc}
\hline Trial & $R T$ (msec) & $S D$ & \% Accuracy & $S D$ \\
\hline NO-P & 942 & 157 & 94.0 & 6.1 \\
NEG-P & 932 & 161 & 94.0 & 7.2 \\
\hline
\end{tabular}

NO-P $=$ no priming; NEG-P $=$ negative priming

were slower to incongruent trials $(895 \mathrm{msec})$ than to congruent trials $(847 \mathrm{msec})[t(17)=5.37, p<.001]$. A significant interference effect was also detected in the terms of performance accuracy (mean interference $=$ $2.3 \%)$, as subjects performed with higher accuracy on congruent trials (97.4\%) compared with incongruent trials $(95.1 \%)[t(17)=2.87, p<.05]$. Descriptive statistics for RT and accuracy data for priming trials are presented in Table 1. Neither in the RT data nor in the accuracy data did NEG-P and NO-P trials differ significantly. Thus, as was anticipated on the basis of the jittered trial timing, no statistically significant generic behavioral NP effects were detected in the current data.

In order to test the prediction of the episodic retrieval model that the ratio between prime-to-probe interval and preprime ISI should affect the temporal discriminability of the prime, and therefore, the degree of NP, we compared NEG-P and NO-P trials with short prime-toprobe intervals and long preprime ISIs with trials with long prime-to-probe intervals and short preprime ISIs. Analyses of simple effects within the NEG-P and NO-P trial types showed that for NEG-P trials, probes with a short prime-to-probe interval and long preprime ISI (mean RT $=948 \mathrm{msec}, S D=168$ ) were significantly longer than to probes displaying the opposite interval ratio (mean $\mathrm{RT}=910 \mathrm{msec}, S D=160)[t(16)=2.22$, $p<.05]$. For NO-P trials, there were no such differences in RTs between trials with a short prime-to-probe interval and long preprime ISI (mean RT $=944 \mathrm{msec}, S D=$ 171) and trials with a long prime-to-probe interval and short preprime ISI (mean RT $=947 \mathrm{msec}, S D=168$ ) $[t(16)=0.28, p>.5]$. However, when submitting these data to a $2 \times 2$ ANOVA with the factors of priming (NEG$\mathrm{P}$ vs. NO-P) and lag ratios (long-short vs. short-long), no significant main or interaction effects were obtained. Therefore, the behavioral data do not offer direct support for an effect of the temporal discriminability of the prime stimulus on the degree of NP.

\section{Imaging Data}

Contrasts between NO-P and NEG-P probe trials revealed two substantial clusters showing increased activation associated with NP (see Figure 2 and Table 2), one located in the right DLPFC, and one located in the right thalamus. The right frontal cluster (57 voxels) was focused in the middle frontal gyrus (GFm) and extended into the superior frontal sulcus (SFs) (Figure 2A). The thalamic activation (30 voxels) was located in the posterior mediodorsal portion of the thalamus (Figure 2B). According to the Talairach Daemon atlas, the peak voxel activation of this cluster would be located in the laterodorsal nucleus (LDn), with the cluster extending into the mediodorsal nucleus (MDn). However, visual inspec-

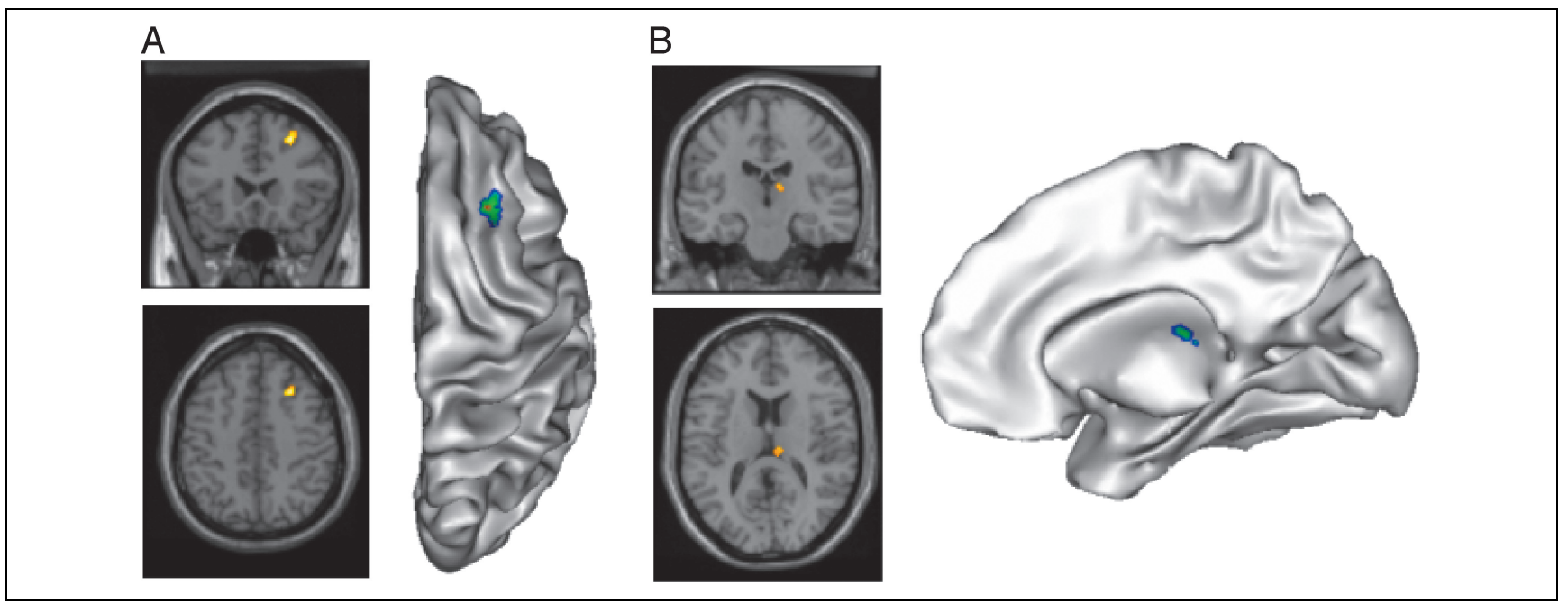

Figure 2. Increased activation in negatively primed probes (NEG-P > NO-P) in (A) the right middle frontal gyrus/superior frontal sulcus, displayed as an activation overlay on a rostral slice (top panel), and an axial slice (bottom panel) of a normalized single-subject T1 scan, and on the white/gray matter border of the dorsal aspect of a partially inflated right hemisphere (right panel) of the same normalized single-subject T1 scan. (B) NP activation in the right mediodorsal thalamus, displayed as an activation overlay on a rostral slice (top panel), and an axial slice (bottom panel) of a normalized single-subject T1 scan, and on the white/gray matter border of the medial aspect of a partially inflated right hemisphere (right panel) of the same normalized single-subject T1 scan. All segmentation and inflation were carried out with Brain VISA software; http://brainvisa.info/index.html. 
Table 2. Brain Regions Displaying Significant Effects of Negative Priming

\begin{tabular}{lllccc}
\hline Region & $B A$ & MNI $(x, y, z)$ & $Z$ score & Cluster & Activation \\
\hline GFm/SFs & 8 & $28,22,46$ & 5.66 & 57 & $\uparrow$ \\
Thalamus & NA & $10,-20,14$ & 4.34 & 30 & $\uparrow$ \\
\hline
\end{tabular}

$\mathrm{BA}=$ Brodmann's area; MNI $(x, y, z)=$ Montreal Neurological Institute coordinates for peak activated voxel in cluster; Cluster $=$ cluster size in voxels, $\uparrow=$ increase in activation; GFm $=$ middle frontal gyrus; SFs $=$ superior frontal sulcus.

tion of the activation overlay, both at the group level and across individual subjects, suggested that the activation was centered on the MDn (see rostral and axial slices displayed in Figure 2B). There were no brain loci that displayed reduced activity in NEG-P compared to NO-P trials. In summary, negatively primed probe trials were associated with increased activation in the right DLPFC and the MDn of the right thalamus.

However, it could be argued that the failure to detect activity in the medial frontal cortex might be due to a lack of sensitivity, or an artifact of our statistical thresholding procedure. Furthermore, the activity found in the right DLPFC could feasibly be related to conflict-related processes, as such responses are not strictly limited to $\mathrm{MPFC}$ regions. In order to dissociate NP processes from conflict-related processes, we therefore carried out additional analyses determining whether (a) the DLPFC region associated with NP displays susceptibility to conflict, and (b) whether conflict-susceptible cortical regions display effects of NP. Regions responsive to conflict were identified by carrying out a region-ofinterest (ROI) analysis within the medial and dorsolateral PFC (BA 6, 8, 9, 24, and 32) for the contrast of incongruent $>$ congruent Stroop trials. At a lenient statistical threshold ( $p<0.01$, uncorrected), two MPFC clusters of activity were detected, one in the ACC $(x=-4, y=34, z=8$; BA 24; 13 voxels) and one in the medial aspect of the superior frontal gyrus $(x=-4$, $y=5, z=55$; BA 6; 17 voxels).

Mean activation values (betas) for congruent and incongruent trials were extracted from these MPFC and the right DLPFC ROIs, normalized, and entered into a $2 \times 2$ ANOVA with the factors ROI (DLPFC vs. MPFC) and Trial congruency (congruent vs. incongruent). Main effects of ROI $[F(1,16)=4.96, p<.05]$ and Congruency $[F(1,16)=3.66, p=.074]$ were qualified by a significant interaction effect $[F(1,16)=4.96, p<.05]$. As can be seen in Figure 3A, although there was an effect of congruency in the MPFC $[t(16)=3.86, p=.001]$, there was no conflict effect evident in the DLPFC $[t(16)=$ $0.40, p>.5]$, and the conflict effect was significantly greater in the MPFC than in the DLPFC $[t(16)=2.23$, $p<.05]$. Therefore, the DLPFC area susceptible to NP could be dissociated from conflict-related processing. Next, in order to assess the degree to which conflictrelated regions might be susceptible to effects of $\mathrm{NP}$, a $2 \times 2$ ANOVA with the factors ROI (DLPFC vs. MPFC) and Priming (NO-P vs. NEG-P) was carried out. Main effects of ROI $[F(1,16)=7.27, p<.05]$ and Priming $[F(1,16)=43.07, p<.001]$ were qualified by a significant interaction effect $[F(1,16)=8.70, p<.01]$. As depicted in Figure 3B, although there was a large effect of NP in the DLPFC $[t(16)=8.48, p<.001]$, there was also a marginally significant effect in the MPFC $[t(16)=$ $2.10, p=.052]$, but the effect of NP was significantly larger in the DLPFC than in the MPFC $[t(16)=2.95$, $p<.01]$. Thus, small effects of NP could be found in conflict-susceptible MPFC regions, but NP effects were significantly larger in the right DLPFC, which in turn was not associated with conflict processing.

Finally, a further corroboration for the dissociation between episodic retrieval processes in the right DLPFC and conflict-related processes in the MPFC could be provided by considering these ROIs' respective responses

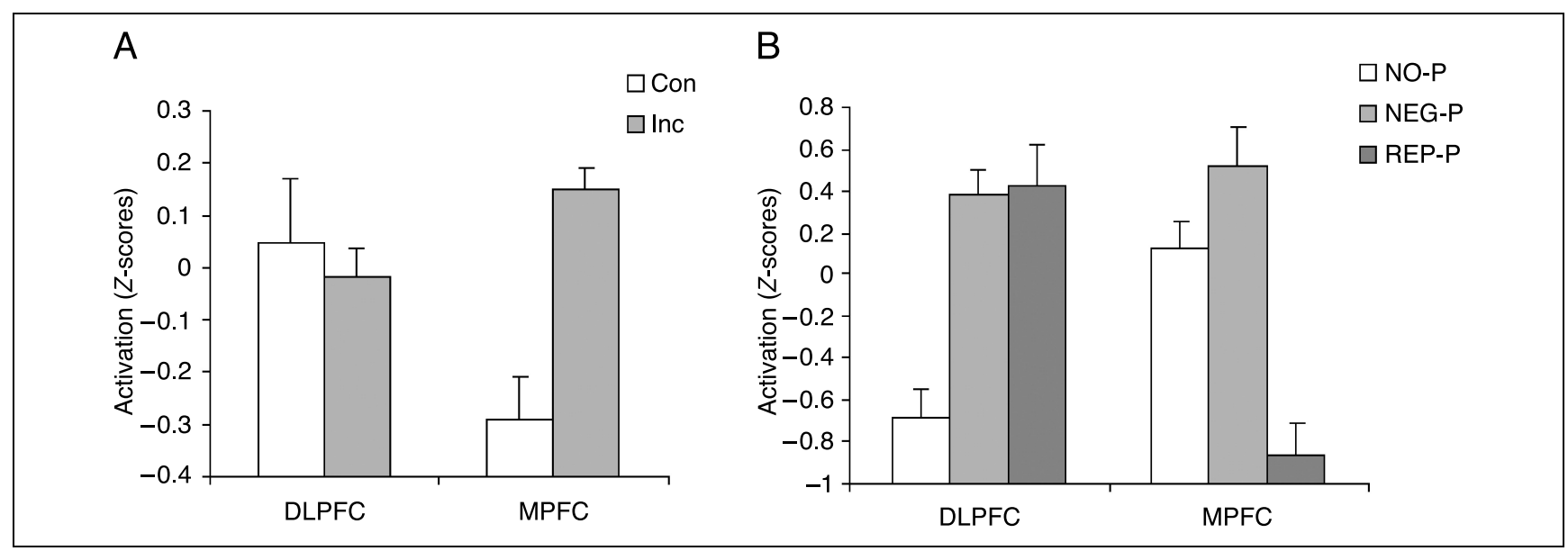

Figure 3. Activation levels (normalized beta values) in the right DLPFC and MPFC ROIs for (A) congruent (Con) versus incongruent (Inc) trials, and (B) no priming (NO-P), negative priming (NEG-P), and repetition priming (REP-P) trials. 
to repetition priming (REP-P) trials, where prime and probe stimuli are identical. These trials are associated with low conflict (mean RT $=720 \mathrm{msec}, S D=110$; faster than NO-P $[t(16)=10.59, p<.001]$ and NEG-P trials $[t(16)=10.38, p<.001])$, but according to the episodic retrieval model should be associated with episodic retrieval processing. Therefore, MPFC responses to REP-P trials should be low, and DLPFC responses should be high. A $2 \times 3$ ANOVA with the factors ROI (DLPFC vs. MPFC) and Priming (NO-P vs. NEG-P vs. REP-P) revealed a main effect of priming $[F(2,32)=13.46, p<.001]$, qualified by a significant interaction effect $[F(2,32)=$ 20.94, $p<.001]$. As displayed in Figure 3B, within the MPFC, REP-P trials were associated with less activation than the NO-P $[t(16)=4.36, p<.001]$ and NEG-P trials $[t(16)=4.99, p<.001]$, whereas in the DLPFC, REP-P trials elicited higher responses than NO-P trials $[t(16)=$ $4.05, p=.001]$, but did not differ from NEG-P trials $[t(16)=0.20, p>.5]$. Thus, the analysis of ROI responses in relation to repetition priming trials has lent further support to the notion that the right DLPFC region implicated in NP is involved in episodic retrieval processes.

\section{Relation between Behavioral and Imaging Data}

Although no significant generic NP effects were detected at the group level, RT differences across subjects can be employed in correlation analyses with individual differences in neural activation within the ROIs identified in the functional imaging results. These correlation analyses serve two purposes. First, they can verify whether DLPFC and MDn activations identified above are directly related to behavioral effects. Secondly, the direction of such correlations can shed light on the functional role played by these structures. On the basis of the episodic retrieval model of $\mathrm{NP}$, it would be expected that the DLPFC activity (putatively reflecting additional episodic retrieval processes) should be positively correlated with RTs on NEG-P trials. Mean activation values (betas) for the two ROIs were extracted from each subject (see fMRI Data Analysis) and entered into bivariate correla- tion analyses with the subjects' mean RTs on NEG-P and NO-P trials. In order to control for global differences in subjects' RTs in these correlations, NO-P and NEG-P RTs were baseline-corrected by subtracting subjects' mean RTs to congruent trials from each of these conditions. One outlier value ( $>2$ SDs from the mean) was excluded from the analysis. The right DLPFC ROI activity displayed a positive association with NEG-P RTs $(r=.48, p=.058)$ (Figure 4A), but no correlation with NO-P RTs $(r=.12$, $n s)$. The MDn ROI activity was negatively correlated with NEG-P RTs $(r=-.53, p<.05)$ (Figure $4 \mathrm{~B})$, but was not correlated with NO-P RTs $(r=.22, n s)$. Thus, activation in both neural foci that were more active in NEG-P as compared to NO-P trials was found to correlate with behavioral performance on NEG-P trials but not on NO-P trials across subjects. These results provide a link between the fMRI data and a behavioral metric of NP, despite the absence of detectable generic NP effects at the group level.

\section{DISCUSSION}

Investigating the neural substrates of NP in a colornaming Stroop task by comparing negatively primed and nonprimed probe stimuli, we found that NP was associated with increased activity in the right DLPFC and the MDn of the right thalamus. This DLPFC activation was shown to be unrelated to conflict-processing, and to display a greater effect of NP than brain regions involved in conflict processing. Neural activity in the DLPFC was positively correlated with individual differences in NEGP RTs, whereas MDn activity displayed a negative correlation. These neurophysiological data support the assumption that NP on target identification tasks stems from the automatic memory retrieval of prior episodes involving the probe stimulus and associated representations (Neill, 1997; Neill \& Valdes, 1992; Neill, Valdes, et al., 1992), and more broadly underline the importance of the interaction between episodic memory and selective attention processes (Logan, 1988).

In neurophysiological terms, the selective inhibition account would predict increased response conflict be-
Figure 4. Correlations between RT on negative priming trials (baseline corrected by subtracting RTs to congruent trials) and activation (betas) in (A) the right DLPFC, and (B) the right mediodorsal thalamus, across subjects.

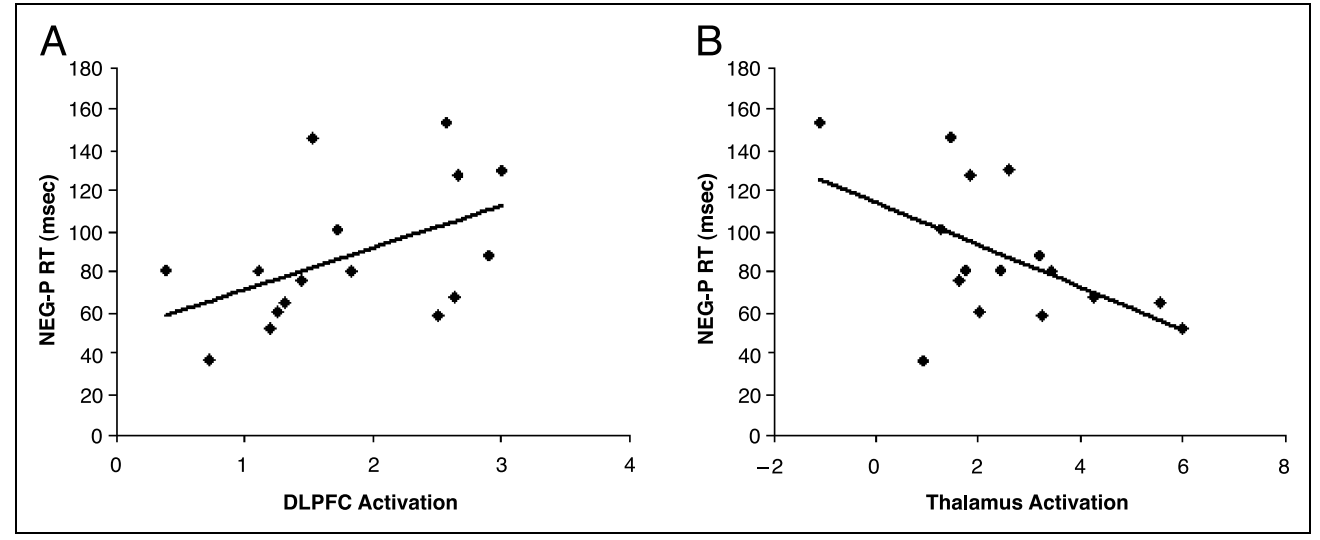


tween color-naming and word-naming processing streams in NP probe trials, resulting in increased MPFC/ACC activation. No significant increase in ACC activity was found in an analysis correcting for whole-brain comparisons. In additional ROI analyses of conflictresponsive regions of the MPFC, it was found that these areas did exhibit a marginal effect of NP. Such an increase in response conflict due to NP can be accounted for by both the selective inhibition model and the episodic retrieval view of NP. However, only the episodic retrieval model predicts additional episodic retrieval processes to occur on NP probe trials, and the current data revealed increased activation of the GFm and SFs in the right DLPFC, a region that has been strongly implicated in memory retrieval processes in previous neuroimaging studies (see Fletcher, Frith, et al., 1997; Buckner et al., 1996). Furthermore, it was demonstrated that this region was not involved in conflict-related processing, and that it displayed a larger effect of NP than the conflict-responsive ROIs.

Right dorsolateral areas, in particular, have been hypothesized to serve the function of monitoring of the appropriateness of episodically retrieved information for the task at hand (Rugg et al., 2003; Henson, Rugg, et al., 2000; Henson, Shallice, et al., 1999; Fletcher, Shallice, et al., 1998; Shallice et al., 1994). This monitoring function of episodic retrieval is thought to be complemented by a cue specification function (Shallice \& Burgess, 1996), suggested to be mediated by the right VLPFC (Rugg et al., 2003; Henson, Rugg, et al., 2000; Henson, Shallice, et al., 1999). If the current contents of episodic retrieval are deemed insufficient or inappropriate by the monitor, it signals the need for additional retrieval processes (Shallice \& Burgess, 1996). Within the framework of this model of episodic memory retrieval, our data can be parsimoniously interpreted as indicating that NP is associated with enhanced monitoring, evaluation, and demand for additional retrieval of episodic memories. These processes are time-consuming, and as a consequence, the responses to negatively primed probes are slowed, either because they directly reflect the outcome of additional retrieval processes, or because they reflect reliance on a slow default generic task algorithm (Neill, 1997; Logan, 1988). This proposal is further supported by the fact that the right DLPFC activation was positively correlated with degree of NEG$\mathrm{P}$ trial RTs across subjects; the more memory retrieval processes are required, the longer it will take to respond to the probe stimulus. In view of these data, we propose that ignored information in displays that allow for perceptual processing of distractor items (see Lavie \& Fox, 2000) is fully encoded and held in episodic memory, and that automatic retrieval of stimulus episodes may aid or interfere with selective attention performance on subsequent trials. Recently, predictions derived from Logan's automatization model with reference to the phenomenon of repetition priming (Logan, 1990) have also found strong support from neuroimaging data (Dobbins, Schnyer, Verfaellie, \& Schacter, 2004).

Our interpretation of the current data does not imply that selective attention does not involve inhibitory processes, but rather that at the time of probe processing, it is episodic retrieval processes that primarily distinguish between negatively primed and nonprimed probe trials. In a recent attempt at integrating the selective inhibition and episodic retrieval views of NP, Tipper (2001) pointed out that inhibitory processes are likely more relevant during and immediately subsequent to the processing of the prime stimulus, whereas retrieval processes would become more relevant at the time of probe presentation. The current data do not conflict with this view, as they only focus on processing of the probe stimuli, where the phenomenon of NP becomes evident. It should be noted, however, that at the time of the prime stimulus presentation, NEG-P trials and NO-P trials are identical, in that both primes are simply incongruent color-word Stroop stimuli. In order to detect neural substrates of suggested inhibitory processes involved in the selective processing of the target stimulus dimension on these trials, these would have to be compared to congruent Stroop trials, but such a comparison necessarily conflates neural correlates of inhibitory processes with those of other concurrent processes, such as response conflict evaluation, response selection, and cognitive control processes (see Egner \& Hirsch, 2005).

An additional neural correlate of NP was found to be increased activation of the right posterior medial thalamus, likely the MDn. The MDn projects richly to lateral prefrontal cortices as part of a dorsolateral prefrontalbasal ganglia-thalamocortical circuit (Alexander, DeLong, \& Strick, 1986). The unexpected finding of MDn activation in relation to NP provides an intriguing segue between our exploration of the basic mechanisms underlying NP in healthy subjects and the clinical domain, where NP has been extensively studied in the context of schizophrenia. On the one hand, it has been documented that schizophrenic patients, as well as healthy volunteers with high scores on self-report measures of schizotypy, exhibit reduced NP effects, that is, faster RTs relative to healthy or low schizotypy controls (MacQueen et al., 2003; Salo, Robertson, \& Nordahl, 1996; Williams, 1995, 1996; Laplante et al., 1992; Beech et al., 1989). On the other hand, much recent research into potential neuroanatomical substrates of schizophrenia has focused on the mediodorsal nucleus of the thalamus, documenting that schizophrenic patients tend to have smaller MDn volumes than matched controls, both in postmortem studies as well as in in vivo structural imaging studies (Kemether et al., 2003; Byne, Buchsbaum, Mattiace, et al., 2002; Byne, Buchsbaum, Kemether, et al., 2001; Popken, Bunney, Potkin, \& Jones, 2000; Young, Manaye, Liang, Hicks, \& German, 2000; Pakkenberg, 1990). Furthermore, metabolic 
activity in the MDn has also been found to be reduced in schizophrenic patients compared to matched controls (Hazlett et al., 1999; Buchsbaum et al., 1996). The current study is to our knowledge the first investigation that demonstrates a direct link between the function of MDn and NP effects in healthy volunteers, and this could provide a crucial insight into the cognitive significance of reduced MDn volumes in schizophrenia. Furthermore, although diminished NP effects in schizophrenics have previously been interpreted exclusively within the framework of impaired inhibitory filtering processes (MacQueen et al., 2003; Salo et al., 1996; Williams, 1995, 1996; Laplante et al., 1992; Beech et al., 1989), our data suggest that these findings may deserve to be re-evaluated in the light of potential episodic memory deficits. It should be noted, however, that MDn activity in the current study was negatively correlated with NEG$\mathrm{P}$ RTs across subjects, whereas schizophrenic patients exhibit less NP and lower MDn volumes (and metabolic activity) than controls, although these two findings have not been documented in the same clinical sample. The relationship between behavioral NP data, MDn function, and disordered cognition in schizophrenia clearly requires further investigation.

In conclusion, negatively primed probe trials on a color-naming Stroop task were associated with increased neural activity in the right DLPFC and thalamic MDn, compared to nonprimed probe trials. The location and response profile of this prefrontal activation strongly suggest that the processing of NP probes involved additional demand on monitoring of episodic retrieval processes, offering neurophysiological support for an episodic retrieval account of NP. Our data imply that ignored stimulus information is fully processed and held in episodic memory, and that automatic episodic retrieval, rather than selective inhibition, of such information affects selective attention performance on subsequent trials. The finding that MDn activation was increased in NP provides an important link between a traditional behavioral measure of supposed inhibitory deficits in schizophrenia (NP) and a suggested anatomical substrate of this condition (MDn), to be explored by future research.

\section{METHODS}

\section{Subjects}

Participants were 17 right-handed, native English-speaking volunteers (mean age $=23.5$ years, age range $=17-$ 33 years, 9 women) who gave written informed consent in accordance with institutional guidelines and the Declaration of Helsinki. All participants had normal or corrected-to-normal vision and were screened by selfreport for previous or current neurological or psychiatric conditions, current medication use, colorblindness, or dyslexia.

\section{Behavioral Paradigm}

The priming Stroop task created for the current study was a four-word color-naming task consisting of the word stimuli BLUE, GREEN, RED, and YELLOW. Each stimulus could be presented in blue, green, red, or yellow hue on a black background, resulting in 16 possible stimuli, four of which were congruent. Stimuli were presented for $1500 \mathrm{msec}$, followed by a central fixation cross. The ISI was jittered between 2000 and $4000 \mathrm{msec}$ (in $500 \mathrm{msec}$ steps) in such a way as to produce a mean ISI of $3000 \mathrm{msec}$ with an approximately Gaussian distribution. Stimuli were presented in 15 blocks of 19 trials each, resulting in 285 trials (34\% congruent and 66\% incongruent) and an overall task length of $16 \mathrm{~min}$, broken down into three runs of equal duration. Of the incongruent trials, $75 \%$ (i.e., $50 \%$ of all trials) were incongruent trials that followed other incongruent trials, and these trial sequences were manipulated so as to result in equal numbers of nopriming trials (NO-P), negative-priming trials (NEG-P), and repetition-priming trials (repetition priming effects were not of primary interest in the current study, and are therefore not addressed in the Results section). Figure 1 shows task timing parameters and example stimuli: In NO-P trials, the target and distractor features of the prime stimulus (the color hue yellow and the word RED, respectively) are unrelated to those of the probe stimulus (the color hue green and the word BLUE). In NEG-P trials, the distractor dimension of the prime trial (the word GREEN) becomes the target dimension of the probe trial (the color hue green). Overall, there were 45 trials in each of these categories. Each block started with a "New Block" instruction presented for $1500 \mathrm{msec}$ and contained a pseudorandom 19-trial stimulus sequence that contained equal numbers of NO-P and NEG-P trials, interspersed with repetition priming trials, congruent trials, and incongruent trials following congruent trials. Subjects were instructed to name the color hue of each stimulus by means of button presses with their left and right middle and index fingers (from left to right: red, blue, green, yellow) as fast as possible while maintaining accuracy. A training period of 5 blocks of the task was administered outside the scanner before the fMRI session. Presentation software (Neurobehavioral Systems, http://nbs.neuro-bs.com) was used to create and deliver the paradigm and record subject responses. The task was presented to the subjects via a back projection onto a screen which could be viewed through a mirror attached to the head coil of the scanner.

\section{fMRI Data Acquisition}

Images were acquired with a GE Signa 1.5-T scanner. Whole-brain functional data were acquired along the 
AC-PC line with a T2*-weighted EPI sequence of 21 contiguous axial slices $(\mathrm{TR}=2000, \mathrm{TE}=40$, flip angle $=$ $60, \mathrm{FoV}=190)$ of $4.5 \mathrm{~mm}$ thickness and $3 \times 3 \mathrm{~mm}$ inplane resolution. The functional data on the Stroop priming task were recorded in three runs of 161 acquisitions each. Structural data were acquired with a highresolution T1-weighted SPGR scan ( $\mathrm{TR}=19, \mathrm{TE}=5$, flip angle $=20, \mathrm{FoV}=220$ ) recording 124 slices at a slice thickness of $1.5 \mathrm{~mm}$ and in-plane resolution of $0.86 \times$ $0.86 \mathrm{~mm}$.

\section{fMRI Data Analysis}

Spatial preprocessing and statistical inference testing were carried out with SPM2 software (Wellcome Department of Cognitive Neurology, University College London, UK, www.fil.ion.ucl.ac.uk/spm/spm2.html). EPI volumes were corrected for differences in acquisition slice-timing, and were spatially realigned to the first volume scanned in the first run. The structural scan was coregistered to a mean image of the realigned functional scans. Then normalization parameters were determined from warping the coregistered structural image to a Montreal Neurological Institute (MNI) template $\mathrm{T} 1 *$ brain, and subsequently, those parameters were applied to the EPI scans, resampling the data at a $2 \mathrm{~mm}^{3}$ voxel size. Finally, the functional images were spatially smoothed with a Gaussian kernel of $6 \times 6 \times$ $9 \mathrm{~mm}$ FWHM (i.e., three times the voxel dimensions as originally acquired). The first three functional volumes were discarded from the analysis.

Within the general linear model framework (Friston et al., 1995), regressors of events, modeled by a standard hemodynamic response function (canonical HRF) and its first temporal derivative (included in order to soak up residual variance due to slight latency variations), were created for each trial type (congruent trials, incongruent trials, NO-P, NEG-P, and repetition trials), as well as for error and posterror trials (see Egner \& Hirsch, 2005). In order to remove low-frequency confounds, data were high-pass filtered (128 sec). An autoregressive function was employed to estimate the temporal autocorrelation in the data and correct degrees of freedom accordingly. For each subject, voxelwise statistical parametric maps (SPMs) were calculated, comparing activation in NEG-P trials to NO-P trials (not including temporal derivative regressors in the contrast), isolating neural correlates of NP. SPMs from each subject were then entered into random-effects analyses at the group level. Statistical significance was determined by applying a whole-brain false discovery rate (Genovese, Lazar, \& Nichols, 2002) of $5 \%$ (i.e., $p \leq 0.05$, corrected) and a cluster-extent threshold of a minimum of 20 contiguous voxels. For correlating BOLD responses from significantly activated regions directly with behavioral measures, mean beta values for functionally defined ROIs (see Results) were extracted from individual subjects' data using Marsbar software (Brett et al., 2002; http:// marsbar.sourceforge.net/).

\section{Acknowledgments}

We thank Graham Jamieson, Chris Summerfield, and two reviewers for helpful comments on this article.

Reprint requests should be sent to Tobias Egner, fMRI Research Center, Columbia University, Neurological Institute Box 108, 710 West 168th Street, New York, NY 10032, USA, or via e-mail: te2111@columbia.edu.

The data reported in this experiment have been deposited with the fMRI Data Center archive (www.fmridc.org). The accession number is $2-2005-118 \mathrm{NJ}$.

\section{REFERENCES}

Alexander, G. E., DeLong, M. R., \& Strick, P. L. (1986). Parallel organization of functionally segregated circuits linking basal ganglia and cortex. Annual Review of Neuroscience, 9, 357-381.

Allport, D. A., Tipper, S. P., \& Chmiel, N. R. J. (1985). Perceptual integration and postcategorical filtering. In M. I. Posner \& O. S. M. Marin (Eds.), Attention and performance XI (pp. 107-132). Hillsdale, NJ: Erlbaum.

Baddeley, A. D. (1976). The psychology of memory. New York: Basic Books.

Beech, A., Powell, T., McWiliam, J., \& Claridge, G. (1989). Evidence of reduced "cognitive inhibition" in schizophrenia. British Journal of Clinical Psychology, 28, 110-116.

Binder, J. R., Rao, S. M., Hammeke, T. A., Frost, J. A., Bandettini, P. A., \& Hyde, J. S. (1994). Effects of stimulus rate on signal response during functional magnetic resonance imaging of auditory cortex. Brain Research, Cognitive Brain Research, 2, 31-38.

Botvinick, M., Nystrom, L. E., Fissell, K., Carter, C. S., \& Cohen, J. D. (1999). Conflict monitoring versus selection-for-action in anterior cingulate cortex. Nature, 402, 179-181.

Botvinick, M. M., Cohen, J. D., \& Carter, C. S. (2004). Conflict monitoring and anterior cingulate cortex: An update. Trends in Cognitive Sciences, 8, 539-546.

Brett, M., Anton, J. L., Valabregue, R., \& Poline, J. B. (2002). Region of interest analysis using an SPM toolbox. Presented at the 8th International Conference on Functional Mapping of the Human Brain. June 2-6, Sendai, Japan. Available on CD-ROM in Neuroimage, 16(2).

Broadbent, D. E. (1958). Perception and communication. London: Pergamon.

Buchsbaum, M. S., Someya, T., Teng, C. Y., Abel, L., Chin, S., Najafi, A., Haier R. J., Wu, J., \& Bunney, W. E., Jr. (1996). PET and MRI of the thalamus in never-medicated patients with schizophrenia. American Journal of Psychiatry, 153, 191-199.

Buckner, R. L., Raichle, M. E., Miezin, F. M., \& Petersen, S. E. (1996). Functional anatomic studies of memory retrieval for auditory words and visual pictures. Journal of Neuroscience, 16, 6219-6235.

Burrock, M. A., Buckner, R. L., Woldorff, M. G., Rosen, B. R., \& Dale, A. M. (1998). Randomized event-related experimental designs allow for extremely rapid presentation rates using functional MRI. NeuroReport, 9, 3735-3739.

Byne, W., Buchsbaum, M. S., Kemether, E., Hazlett, E. A., Shinwari, A., Mitropoulou, V., \& Siever, L. J. (2001). Magnetic resonance imaging of the thalamic mediodorsal 
nucleus and pulvinar in schizophrenia and schizotypal personality disorder. Archives in General Psychiatry, 58, 133-140.

Byne, W., Buchsbaum, M. S., Mattiace, L. A., Hazlett, E. A., Kemether, E., Elhakem, S. L., Purohit, D. P., Haroutunian, V., \& Jones, L. (2002). Postmortem assessment of thalamic nuclear volumes in subjects with schizophrenia. American Journal of Psychiatry, 159, 59-65.

Carter, C. S., Macdonald, A. M., Botvinick, M., Ross, L. L., Stenger, V. A., Noll, D., \& Cohen, J. D. (2000). Parsing executive processes: Strategic vs. Evaluative functions of the anterior cingulate cortex. Proceedings of the National Academy of Sciences, U.S.A., 97, 1944-1948.

Casey, B. J., Thomas, K. M., Welsh, T. F., Badgaiyan, R. D., Eccard, C. H., Jennings, J. R., \& Crone, E. A. (2000). Dissociation of response conflict, attentional selection, and expectancy with functional magnetic resonance imaging. Proceedings of the National Academy of Sciences, U.S.A., 97, 8728-8733.

Cohen, J. D., Dunbar, K., \& McClelland, J. L. (1990). On the control of automatic processes: A parallel distributed processing account of the stroop effect. Psychological Review, 97, 332-361.

Dale, A. M. (1999). Optimal experimental design for event-related fMRI. Human Brain Mapping, 8, 109-114.

Dalrymple-Alford, E. C., \& Budayr, B. (1966). Examination of some aspects of the Stroop color-word test. Perceptual and Motor Skills, 23, 1211-1214.

Deutsch, J. A., \& Deutsch, D. (1963). Attention: Some theoretical considerations. Psychological Review, 70, 80-90.

Dobbins, I. G., Schnyer, D. M., Verfaellie, M., \& Schacter, D. L. (2004). Cortical activity reductions during repetition priming can result from rapid response learning. Nature, 428, 316-319.

Driver, J., \& Baylis, G. C. (1993). Cross-modal negative priming and interference in selective attention. Bulletin of the Psychonomic Society, 31, 45-48.

Egner, T., \& Hirsch, J. (2005). The neural correlates and functional integration of cognitive control in a stroop task. Neuroimage, 24, 539-547.

Fletcher, P. C., Frith, C. D., \& Rugg, M. D. (1997). The functional neuroanatomy of episodic memory. Trends in Neurosciences, 20, 213-218.

Fletcher, P. C., Shallice, T., \& Dolan, R. J. (1998). The functional roles of prefrontal cortex in episodic memory: I. Encoding. Brain, 121(Pt 7), 1239-1248.

Fox, E. (1995). Negative priming from ignored distractors in visual selection: A review. Psychonomic Bulletin Review, 2, $145-173$.

Friston, K. J., Holmes, A. P., Worsley, K. J., Poline, J.-B., Frith, C. D., \& Frackowiak, R. S. J. (1995). Statistical parametric maps in functional imaging: A general linear approach. Human Brain Mapping, 2, 189-210.

Genovese, C. R., Lazar, N. A., \& Nichols, T. (2002). Thresholding of statistical maps in functional neuroimaging using the false discovery rate. Neuroimage, 15, 870-878.

Hazlett, E. A., Buchsbaum, M. S., Byne, W., Wei, T. C., Spiegel-Cohen, J., Geneve, C., Kinderlehrer, R., Haznedar, M. M., Shihabuddin, L., \& Siever, L. J. (1999). Three-dimensional analysis with MRI and PET of the size, shape, and function of the thalamus in the schizophrenia spectrum. American Journal of Psychiatry, 156, 1190-1199.

Henson, R. N., Rugg, M. D., Shallice, T., \& Dolan, R. J. (2000). Confidence in recognition memory for words:
Dissociating right prefrontal roles in episodic retrieval Journal of Cognitive Neuroscience, 12, 913-923.

Henson, R. N., Shallice, T., \& Dolan, R. J. (1999). Right prefrontal cortex and episodic memory retrieval: A functional mri test of the monitoring hypothesis. Brain, 122(Pt 7), 1367-1381.

Houghton, G., \& Tipper, S. P. (1994). A model of inhibitory mechanisms in selective attention. In D. Dagenbach \& T. Carr (Eds.), Inbibitory mechanisms in attention, memory and language (pp. 53-112). San Diego: Academic Press.

Houghton, G., \& Tipper, S. P. (1996). Inhibitory mechanisms of neural and cognitive control: Applications to selective attention and sequential action. Brain and Cognition, 30, 20-43.

Kemether, E. M., Buchsbaum, M. S., Byne, W., Hazlett, E. A., Haznedar, M., Brickman, A. M., Platholi, J., \& Bloom, R. (2003). Magnetic resonance imaging of mediodorsal, pulvinar, and centromedian nuclei of the thalamus in patients with schizophrenia. Archives in General Psychiatry, 60, 983-991.

Kerns, J. G., Cohen, J. D., MacDonald, A. W., 3rd, Cho, R. Y., Stenger, V. A., \& Carter, C. S. (2004). Anterior cingulate conflict monitoring and adjustments in control. Science, 303, 1023-1026.

Laplante, L., Everett, J., \& Thomas, J. (1992). Inhibition through negative priming with Stroop stimuli in schizophrenia. British Journal of Clinical Psychology, 31, 307-326.

Lavie, N., \& Fox, E. (2000). The role of perceptual load in negative priming. Journal of Experimental Psychology: Human Perception and Performance, 26, 1038-1052.

Logan, G. D. (1988). Toward an instance theory of automatization. Psychological Review, 95, 492-527.

Logan, G. D. (1990). Repetition priming and automaticity: Common underlying mechanisms? Cognitive Psychology, 22, 1-35.

MacLeod, C. M. (1991). Half a century of research on the stroop effect: An integrative review. Psychological Bulletin, 109, 163-203.

MacQueen, G. M., Galway, T., Goldberg, J. O., \& Tipper, S. P. (2003). Impaired distractor inhibition in patients with schizophrenia on a negative priming task. Psychological Medicine, 33, 121-129.

May, C. P., Kane, M. J., \& Hasher, L. (1995). Determinants of negative priming. Psychological Bulletin, 118, 35-54.

Mayr, S., Niedeggen, M., Buchner, A., \& Pietrowsky, R. (2003). ERP correlates of auditory negative priming. Cognition, 90, B11-21.

Neill, W. T. (1977). Inhibitory and facilitatory processes in selective attention. Journal of Experimental Psychology: Human Perception and Performance, 3, 444-450.

Neill, W. T. (1979). Switching attention within and between categories: Evidence for intracategory inhibition. Memory E Cognition, 7, 283-290.

Neill, W. T. (1997). Episodic retrieval in negative priming and repetition priming. Journal of Experimental Psychology: Learning, Memory, and Cognition, 23, 1291-1305.

Neill, W. T., Lissner, L. S., \& Beck, J. L. (1990). Negative priming in same-different matching: Further evidence for a central locus of inhibition. Perception \& Psychophysics, 48, 398-400.

Neill, W. T., \& Valdes, L. A. (1992). Persistence of negative priming: Steady state or decay? Journal of Experimental Psychology: Learning, Memory, and Cognition, 18, 565-576.

Neill, W. T., Valdes, L. A., Terry, K. M., \& Gorfein, D. S. (1992). Persistence of negative priming: II. Evidence for episodic 
trace retrieval. Journal of Experimental Psychology: Learning, Memory, and Cognition, 18, 993-1000.

Neill, W. T., \& Westberry, R. L. (1987). Selective attention and the suppression of cognitive noise. Journal of Experimental Psychology: Learning, Memory, and Cognition, 13, 327-334.

Norman, D. A. (1968). Toward a theory of memory and attention. Psychological Review, 75, 522-536.

Pakkenberg, B. (1990). Pronounced reduction of total neuron number in mediodorsal thalamic nucleus and nucleus accumbens in schizophrenics. Archives of General Psychiatry, 47, 1023-1028.

Popken, G. J., Bunney, W. E., Jr., Potkin, S. G., \& Jones, E. G. (2000). Subnucleus-specific loss of neurons in medial thalamus of schizophrenics. Proceedings of the National Academy of Sciences, U.S.A., 97, 9276-9280.

Rugg, M. D., Henson, R. N., \& Robb, W. G. (2003). Neural correlates of retrieval processing in the prefrontal cortex during recognition and exclusion tasks. Neuropsychologia, 41, 40-52.

Rugg, M. D., \& Nagy, M. E. (1989). Event-related potentials and recognition memory for words. Electroencephalography and Clinical Neurophysiology, 72, 395-406.

Salo, R., Robertson, L. C., \& Nordahl, T. E. (1996). Normal sustained effects of selective attention are absent in schizophrenic patients withdrawn from medication. Psychiatry Research, 62, 121-130.

Shallice, T., \& Burgess, P. (1996). The domain of supervisory processes and temporal organization of behaviour. Philosophical Transactions of the Royal Society of London, Series B. Biological Sciences, 351, 1405-1411; discussion 1411-1402.

Shallice, T., Fletcher, P., Frith, C. D., Grasby, P., Frackowiak, R. S. J., \& Dolan, R. J. (1994). Brain regions associated with acquisition and retrieval of episodic working memory. Nature, 368, 633-635.

Steel, C., Haworth, E. J., Peters, E., Hemsley, D. R., Sharma, T., Gray, J. A., Pickering, A., Gregory, L., Simmons, A., Bullmore, E. T., \& Williams, S. C. (2001). Neuroimaging correlates of negative priming. NeuroReport, 12, 3619-3624.

Stroop, J. R. (1935). Studies of interference in serial verbal reactions. Journal of Experimental Psychology, 18, 643-662.

Tipper, S. P. (1985). The negative priming effect: Inhibitory priming by ignored objects. Quarterly Journal of Experimental Psychology A, 37, 571-590.

Tipper, S. P. (2001). Does negative priming reflect inhibitory mechanisms? A review and integration of conflicting views. Quarterly Journal of Experimental Psychology, 54A, 321-343.

Tipper, S. P., \& Cranston, M. (1985). Selective attention and priming: Inhibitory and facilitatory effects of ignored primes. Quarterly Journal of Experimental Psychology A, 37, 591-611.

Tipper, S. P., \& Driver, J. (1988). Negative priming between pictures and words in a selective attention task: Evidence for semantic processing of ignored stimuli. Memory $\mathcal{E}$ Cognition, 16, 64-70.

Tipper, S. P., MacQueen, G. M., \& Brehaut, J. C. (1988). Negative priming between response modalities: Evidence for the central locus of inhibition in selective attention. Perception \& Psychophysics, 43, 45-52.

Treisman, A. M. (1969). Strategies and models of selective attention. Psychological Review, 76, 282-299.

Ullsperger, M., \& von Cramon, D. Y. (2001). Subprocesses of performance monitoring: A dissociation of error processing and response competition revealed by event-related fMRI and ERPs. Neuroimage, 14, 1387-1401.

Vazquez, A. L., \& Noll, D. C. (1998). Nonlinear aspects of the BOLD response in functional MRI. Neuroimage, 7 , 108-118.

Wager, T. D., \& Nichols, T. E. (2003). Optimization of experimental design in fMRI: A general framework using a genetic algorithm. Neuroimage, 18, 293-309.

Williams, L. M. (1995). Further evidence for a multidimensional personality disposition to schizophrenia in terms of cognitive inhibition. British Journal of Clinical Psychology, 34, 193-213.

Williams, L. M. (1996). Cognitive inhibition and schizophrenic symptom subgroups. Schizophrenia Bulletin, 22, 139-151.

Yee, P. L. (1991). Semantic inhibition of ignored words during a figure classification task. Quarterly Journal of Experimental Psychology A, 43, 127-153.

Young, K. A., Manaye, K. F., Liang, C., Hicks, P. B., \& German, D. C. (2000). Reduced number of mediodorsal and anterior thalamic neurons in schizophrenia. Biological Psychiatry, 47, 944-953. 